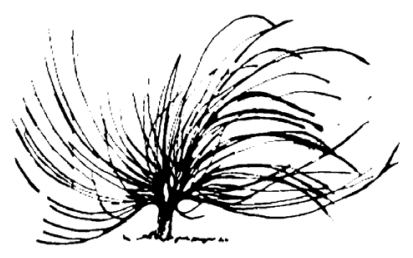

\title{
Perfil de salida de la Escuela de Arte Escénico, reflexionando sobre su misión
}

\author{
Wendy Hall Fernández ${ }^{1}$ \\ Universidad Nacional \\ Costa Rica \\ wendy.hall.fernandez@una.cr
}

\begin{abstract}
Resumen
En el siguiente artículo, se presenta un proceso de investigación con la intención pedagógica de ahondar en el perfil de salida de la población de personas graduadas de la Escuela de Arte Escénico de la Universidad Nacional de Costa Rica (EAE), como primer acercamiento a reflexionar y analizar la misión de la EAE respecto a la formación de profesionales y los roles que pueden desempeñar al egresar o graduarse. Por tanto, la investigación se centra en determinar la situación laboral de la población egresada y graduada. Esta se desarrolló durante el periodo de 2017-2018 y se sistematiza en el 2019, dentro del marco del Proyecto Pre-Carrera de la misma escuela. El proceso se aplica de manera participativa con personas que son estudiantes actuales, egresadas y funcionarias de la EAE, con lo cual se pretende dar continuidad a un proceso de reflexión sobre la pedagogía teatral que surgió a partir de 2013 y que se mantiene hasta la actualidad.
\end{abstract}

Palabras claves: teatro, pedagogía teatral, pedagogía.

\section{(C) $\odot \Theta \Theta$}

Recibido: 31 de agosto de 2019. Aprobado: 2 de setiembre de 2020.

http://dx.doi.org/10.15359/rep.16-1.9

1 Artista escénica, académica en la Escuela de Arte Escénico, graduada de la Maestría en Pedagogía con énfasis en Diversidad en los Procesos Educativos de la Universidad Nacional de Costa Rica. https://orcid.org/0000-0002-2576-3464 


\begin{abstract}
This article describes a research process with the pedagogical aim of delving into the exit profile of graduates from the School of Performing Arts of Universidad Nacional, Costa Rica as a first approach to reflecting on and analyzing the school's mission statement in terms of training professionals and the roles they can play after completing the major or graduating from it. Therefore, this research is focused on determining the employment situation of the population who completed the major or graduated from it. The research was developed during the period of 2017-2018, and it was systematized in 2019 as part of the pre-major project of the school. This process was carried out in a participatory way with the people who are current students, graduates, and staff from the School of Performing Arts, thereby following a reflection process on theater pedagogy which began in 2013 and continues to the present.
\end{abstract}

Keywords: theater, theater pedagogy, pedagogy

\title{
Introducción
}

Desde el año 2014, inicia un plan piloto de un posible proyecto denominado Pre-Carrera de la Escuela de Arte Escénico (PCEAE), el cual nace con varios objetivos, entre ellos acercar a las personas interesadas en ingresar a la EAE al arte escénico, contribuyendo de esa manera con la misión de la misma escuela. Lo anterior sucede dado que durante los talleres libres de actuación ofrecidos por el Programa Teatro Aplicado (PTA), los participantes solicitaban mayor información y, principalmente, mencionaban la necesidad de más y mejores espacios que pudieran acercarles al Arte Escénico.

Además, cabe destacar que muchas de esas personas que estaban en dichos cursos realizaron también el proceso de admisión de la EAE. Al iniciar un proceso de un proyecto de docencia, es necesario que paralelamente se desarrolle un trabajo de investigación, por lo que, si la meta era acercar a la población y contribuir con la visión de la escuela, es fundamental también realizar ciertas preguntas, entrar a dialogar con ellas. 
Algunos de los cuestionamientos iniciales fueron: ¿para qué? ¿cómo? y ¿por qué contribuir y acercar? Sin embargo, luego de mucha reflexión y análisis, se consideró que el proceso del Proyecto Pre-Carrera es también sobre y para la pedagogía teatral, en este caso en el nivel superior. Según Vásquez (2009), la pedagogía teatral se define como:

...un conjunto de conocimientos -saberes- haceres especializados y articulados interdisciplinariamente, que dictan los modos de pensar-reflexionar, planificar-programar, accionar-activar, observar-estudiar, evaluar procesos, problematizar-conceptualizar y producir-construir competencias profesionales de la enseñanza y el aprendizaje de los sujetos, objetos, agentes y medios, en el ámbito de la educación artística teatral, y en el contexto sociocultural en el que este se desarrolla. (p. 66)

Responder a las preguntas antes mencionadas condujo a la investigación a realizar un estudio sobre el perfil de entrada del estudiantado de la EAE, por ejemplo, surgieron interrogantes como: ¿a cuáles personas se quiere acercar? y ¿cuáles personas quieren acercarse a la EAE? "En la enseñanza se han olvidado de las preguntas, tanto la persona docente, como la estudiantil las han olvidado y, en mi opinión, todo conocimiento comienza por la pregunta" (Freire y Faúndez, 2013, p. 69).

A partir de allí se genera un proceso de análisis de los datos encontrados en los formularios que se realiza durante el proceso de admisión, intentando identificar a esas personas interesadas en ingresar, acercándose a otro perfil, que en este caso sería el de salida. Una persona que quiere acercarse a la EAE lo hace porque quiere llegar a ser esto o lo otro cuando salga de la escuela.

Se definieron tres propósitos para el Proyecto Pre-Carrera: a) reconocer la necesidad de contribuir y reflexionar con la EAE en esa formación de profesionales, posibilitando un espacio de transición, b) reflexionar sobre quiénes son esas personas que desean ingresar, perfil de entrada y por tanto c) considerar las que salen, perfil de salida. Ninguno de los propósitos fue en detrimento del otro, sino que han generado un material definitorio para la continuidad del proyecto y principalmente, han resumido un viaje pedagógico que demuestra un crecimiento en la pedagogía teatral costarricense en el nivel superior. 


\section{Recolección de datos perfil de salida: ¿qué datos se recolectan? y principalmente ¿cuáles son sus razones?}

Durante el proceso de investigación para la creación del proyecto Pre-Carrera de la Escuela de Arte Escénico, el cual actualmente se encuentra activo, se realizaron varias entrevistas, una de ellas al director de la escuela, Gabrio Zappelli Cerri, así como también, entrevistas específicas a docentes y a estudiantes, todas estas durante el año 2014 y con la intención de justificar la necesidad de avalar un proyecto como el Pre-Carrera.

En ese mismo año, la académica Dora Cerdas Bokhan realizó un pequeño análisis sobre los planes de estudio (Cerdas, 2014), con la sistematización de las entrevistas, el estudio del libro de colección, entre otros aspectos, como por ejemplo la búsqueda de material teórico sobre la pedagogía teatral en el nivel superior costarricense, posibilitaron la necesidad de darle continuidad a la creación del proyecto, así como agregarle el ingrediente de la investigación de la pedagogía teatral, dado que existe una ausencia en la investigación pedagógica en materia de la educación en arte escénico en el nivel superior.

La búsqueda de respuestas llevó a cuestionarse sobre cuáles serían las preguntas indicadas; para ello, se entregó la información a diversas personas para comentar y realizar propuestas de preguntas; algunas de las que colaboraron fueron la señora Vera Ramírez Briceño, productora actual de la EAE, quien en su momento fungía como subdirectora, y el presidente de la Asociación de Estudiantes, el cual permaneció durante el proceso de recolección de información en el año 2016, y actualmente es Licenciado en Arte Escénico.

Como no se ha realizado una investigación relacionada con la educación teatral en el nivel superior, se partió de lo concreto, no apresurar en valorar el fondo de la educación, sino hacer consulta del estado de la situación. Existen dos tesis sobre las escuelas de teatro en Costa Rica, pero ninguna aborda la pedagogía, así como investigaciones sobre pedagogía, pero ninguna sobre educación superior.

En el nivel metodológico, se decidió trabajar con un formulario en línea, entrevistas abiertas y grupos focales a personas graduadas y egresadas de la EAE, egresadas dado que en la escuela existe un porcentaje de individuos que no termina el bachillerato; sin embargo, según 
datos del formulario, $7.5 \%$ de las personas graduadas de bachillerato y $20.9 \%$ egresadas de licenciatura ejercen el arte escénico.

Luego se desarrolló la sistematización de los instrumentos; a continuación, se detalla un poco el proceso: junto con la estudiante asistente Karla Barquero Mata se realizó un formulario que comprendía doce preguntas: tres de información personal para contextualizar la población, nueve sobre la investigación específica del perfil de salida y otras dos eran de selección en las que se pedía la profundización de la respuesta.

Las preguntas se enfocaban en si se estaba ejerciendo teatro en la actualidad, en qué área del arte escénico específicamente, en qué área se ha ejercido en el nivel laboral y en el nivel de la actualización profesional. Se decidió, además, aprovechar la encuesta para consultar sobre año de salida y estudios en otras áreas o carreras.

En total respondieron 70 personas, sumando a estas los datos de quienes terminaron la licenciatura desde los inicios de la EAE, los cuales se encontraban ya registrados en la escuela y contienen la información de los respectivos procesos de investigación de cada estudiante.

La encuesta permaneció abierta por cinco meses; durante esa época se difundió en diversas redes sociales, lo cual le dio una limitación y una ventaja, al menos. La ventaja: llegar a las personas que efectivamente, están ejerciendo teatro; la limitación: no llegar a personas que decidieron por diversas razones dejar de estar en el gremio teatral.

Las redes sociales utilizadas fueron Facebook e Instagram, así como el correo electrónico, por medio de un afiche promocional que invitaba a la consulta. La limitación cada vez fue más evidente, se pudo obtener datos de las personas que están realmente ejerciendo teatro o bien están cercanas aún al gremio artístico.

La pregunta ¿qué estamos haciendo las personas egresadas de la EAE? se convirtió en ¿qué estamos haciendo las personas egresadas de la EAE cercanas al teatro? Por tanto, se consideró oportuno darle continuidad a otro proceso de investigación que quedará pendiente, ¿cómo obtener datos de las personas que se alejaron del gremio? Para tener los datos de la cantidad de personas que no se lograron contactar se debe buscar la cantidad de personas matriculadas durante los años de investigación. 
$\mathrm{Al}$ tener los datos cuantitativos, se compartieron las cifras para que en colectivo se realizara una lectura inicial de estas, así que se llevó a cabo una mesa de diálogo, invitando a las personas que participaron en la encuesta y a cuatro de manera específica para que conversaran como panelistas.

Mesa de diálogo 1: compartir los resultados de la encuesta: ¿qué estamos haciendo las personas egresadas y graduadas de la EAE?

Leer datos es también subjetivo, dado que intervienen en este proceso la historia de vida, la escolaridad, la profesión e incluso las actualizaciones profesionales de la persona que los lee, que dialoga con ellos; por más objetiva que sea, les dará una razón de ser desde su individualidad. Fue necesario para las dos coinvestigadoras abrazar más lecturas para así obtener más información, más subjetividades. Por ello, se consideró oportuno realizar un compartir con algunas personas invitadas.

El sábado 4 de mayo de 2017, junto a Tatiana Chaves Araya, actual coordinadora del área artística de la Municipalidad de San José, egresada de licenciatura de la Escuela de Arte Escénico; Reinaldo Amién Gutiérrez y Paola González Vargas, personas egresadas también de la licenciatura y que además actualmente trabajan como docentes en la EAE, y Alicia Riba Hernández, egresada de bachillerato, modelo de la Escuela de Bellas Artes de la Universidad de Costa Rica, se inició un diálogo con el propósito de leer los datos recogidos en el nivel cuantitativo de la encuesta realizada.

Desarrollar el pensamiento colectivo por medio del diálogo posibilita interpretaciones colectivas, "el pensamiento es la materia prima de los momentos de conversación, de discusión y debate. La calidad del razonamiento afecta a la calidad de la discusión. La discusión es, en buena medida, pensamiento en interacción" (Medina y Cembranos 2003, p. 29).

Las preguntas generadoras fueron discutidas y reflexionadas en colectivo por medio de las personas coinvestigadoras:

- ¿En cuáles espacios ha encontrado mayores ofertas/oportunidades laborales desde que finalizó la carrera de Arte Escénico?

- ¿En cuáles campos se sintió como una persona forzada a capacitarse una vez finalizada la carrera? 
- ¿Según nuestro contexto actual costarricense considera que al gremio teatral le beneficia una actualización general en varios campos o especializarse en uno concreto?

Se reconoce que el solo hecho de realizar preguntas generadoras dirige la conversación; sin embargo, se realizaron algunas adicionales como motor para esta. La actividad fue intensa, dos horas de diálogo entre personas invitadas, quienes con gran compromiso quisieron escuchar y tomar protagonismos para así reflexionar en colectivo los resultados. Sucedió lo que se esperaba, una mesa de diálogo que posibilitó algunos discursos de pensamiento crítico y cuestionamientos de accionares del gremio teatral.

Algunas de las lecturas realizadas a los resultados por las personas participantes de las mesas ya son conocidas, han sido dichas durante conversaciones entre colegas en reuniones sociales u otros espacios; el gremio teatral es difícil, exigente, sensible a comentarios y desordenado, se incluyó también los adjetivos de disperso y desligado.

Se mencionaron además, detalles como la dificultad de ser y estar en un país tercermundista, en donde, aunque comparado con otros centroamericanos, se tienen muchas ventajas y privilegios, también varias limitaciones; por ello, corresponde hacer un poco de todo, idea con la cual nació durante la conversación un concepto quizá muy conocido en el contexto teatral costarricense: la persona "todóloga". Entonces, como menciona Araque (2009), se deben tomar en cuenta otros dilemas que no se pueden dejar de lado:

...debería exigirse otras competencias del egresado, y ellas no serían las aceptadas por el sentido común o por el Estado que sólo ve la posibilidad de formación en el sentido de cumplir las cuotas sociales, en la dirección de cubrir los mercados. Ese es otro verdadero dilema. (p. 61)

La persona "todóloga" es la que tiene que hacer de todo por ausencia de presupuesto y recursos, esto se da principalmente en las agrupaciones independientes; sin embargo, en la institucionalidad sucede. Por ello, se consideró que es la necesidad la que genera las actualizaciones profesionales técnicas, universitarias, de grado o posgrado y en cursos y talleres libres. Es la necesidad de hacer teatro la que 
ha generado que las personas del gremio realicen actividades propias de otras disciplinas, como lo son la enseñanza, la contabilidad, la producción, la generación de públicos, entre otras actividades.

Pero esa necesidad no es solamente negativa, en ocasiones posibilita el descubrir otras pasiones, otras potencialidades y talentos en las personas, aquellas que además consideran que esa necesidad debe ser, con un compromiso ético y por tanto investigada. Es incluso lo que hace que personas decidan especializarse en otras áreas y que sea luego otro perfil de trabajo.

En colectivo se detectó un problema importante y es cuando, por el contrario, la persona decide dedicarse a la enseñanza, ya sea por necesidad o interés, situación que representa el porcentaje mayor de respuesta a la pregunta ¿qué estamos haciendo las personas graduadas o egresadas de la EAE? Sin embargo, a la hora de comparar con las actualizaciones, se aprecia que no existe en enseñanza, educación o pedagogía; por tanto, ejercen con lo estudiado/ofrecido en la carrera de Arte Escénico, reproduciendo lo aprendido y aprehendido.

Para este momento el proceso de investigación se mantenía dentro del proyecto Pre-Carrera que fue creado y avalado como formal dentro de la EAE; participó Sara Astica en la Cátedra de la Escuela de Arte Escénico denominada La pedagogía teatral y sus múltiples alcances, por medio de un avance de la investigación en el 2016. El siguiente apartado detalla parte de los resultados sin explicaciones mayores, con la intención de que sean objetivos a la mirada de quien lee $\mathrm{y}$, de esa manera, posibilitar nuevas interpretaciones.

\section{Datos cuantitativos y cualitativos seleccionados para realizar la lec- tura a la pregunta: ¿qué estamos haciendo las personas graduadas y egresadas de la EAE?}

Luego de ordenar todas las respuestas del formulario, se seleccionaron aquellas más recurrentes, con mayores porcentajes, para realizar una lectura generalizada y, por ende, definir categorías. Se contemplan los datos generales, pero esto no quiere decir que no exista lo específico; por ejemplo, personas graduadas que han tenido los privilegios económicos y académicos para salir del país y beneficiarse de estudios que les han posibilitado otras opciones; egresadas que decidieron por sus historias de vida no continuar con el arte escénico y otras que, gracias a su pensamiento complejo, dispusieron acercarse a áreas diversas 
de las artes o de las ciencias, como las exactas o sociales. Para este escrito los datos seleccionados son:

Campo de desarrollo profesional

1. $\quad 39.6 \%$ en docencia

2. $32.1 \%$ en teatro independiente

3. $20.8 \%$ otro (investigación académica y teatro privado)

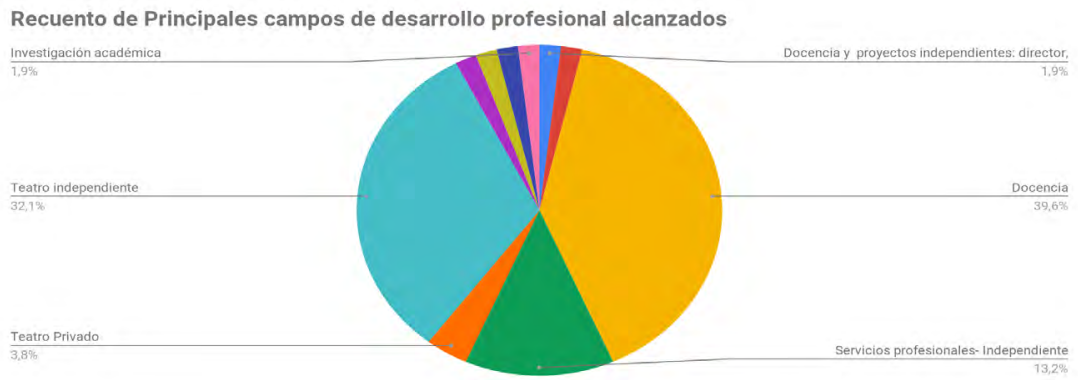

Figura 1. Recuento de principales campos de desarrollo profesional alcanzados

Nota: elaboración propia.

Estudió otra carrera, además de teatro

1. $25.4 \%$ Sí

2. $74.6 \%$ No

Actualización formal o no formal

1. $\quad 13.4 \%$ Formal

2. $52.2 \%$ No formal

3. $\quad 17.9 \%$ Ambas

4. $\quad 16.4 \%$ No contestó

Terminó licenciatura, bachillerato o nivel

1. $\quad 49.3 \%$ Bachillerato

2. $22.4 \%$ Licenciatura

3. $\quad 16.4 \%$ Proceso de Licenciatura

4. $\quad 11.9 \%$ No terminó la carrera

Quiere decir que las personas graduadas de la Escuela de Arte Escénico en su mayoría son bachilleres que se actualizan de manera 
no formal y enseñan teatro, paralelo a sus trabajos independientes. De acuerdo con estadísticas estudiantiles de la Universidad Nacional, de su Departamento de Registro de la Vicerrectoría de Docencia, del año 2007 al 2018 se han graduado 133 personas de la Escuela de Arte Escénico, en su totalidad; de ellas 118 terminaron el bachillerato y 33, la licenciatura. En este estudio también se refleja que son más las mujeres matriculadas y, por tanto, las graduadas, ya que 71 terminaron el bachillerato y 18 , la licenciatura (Universidad Nacional, 2019).

En relación con el perfil de entrada, existen datos de las investigaciones anteriores que no se pueden obviar; aunque las personas a las cuales se les hizo el análisis de perfil de salida no son las mismas a las que se les realizó el estudio de perfil de entrada, son valores que pueden colaborar al momento de hacer una lectura del perfil de salida.

Por ejemplo, durante el periodo comprendido entre el 2007 y el 2019 han matriculado de primer ingreso 275 personas; en relación con este dato, se podría mencionar que la matrícula de la EAE es principalmente de mujeres que iniciaron su proceso universitario jóvenes de entre 17 y 19 años y prioriza la zona urbana, dados los siguientes porcentajes también tomados de estadísticas estudiantiles:

1. $60 \%$ Mujeres

2. $\quad 45.7 \% 17-19$ años

3. $\quad 84.25 \%$ Zona Urbana (Universidad Nacional, 2020)

\section{Relación de comentarios y posibles lecturas entre personas es- tudiantes y profesionales invitadas a raíz de los resultados de la encuesta}

Existen razones políticas, sociales, entre otras que podríamos profundizar, por las cuales se toman las decisiones laborales de las personas profesionales egresadas de la Escuela de Arte Escénico y específicamente del teatro; sin embargo, se tomarán en cuenta factores que fueron mencionados en la mesa de diálogo.

Estos tienen que ver con la memoria histórica que posee el gremio teatral costarricense, así como con las instituciones que posibilitan el estudio del arte escénico, dramático y otros detalles que tienen relación tanto con las corrientes pedagógicas utilizadas o no, por las instancias de educación superior, como también las oportunidades que se generan en el momento en el que las personas están cursando la carrera. Estas causas, no menos importantes una de la otra, son necesarias para valorar 
lo que están haciendo quienes egresan de la EAE. Como se mencionó anteriormente, si la persona graduada y egresada de Arte Escénico se dedica a la enseñanza por necesidad, ¿implica que no lo hace por vocación?; esto validaría las palabras de las personas invitadas en la mesa de diálogo:

...el grueso, refiriéndose a las personas egresadas, se dedica a la docencia pero entonces hay un 3\% con una buena formación y eso es terrible, por ejemplo a mí me llamaron a dar clases y dije ok, sí, yo voy a echar a perder, voy a echar a perder bien, sí eso dije, porque es una gran responsabilidad, entonces me metí a estudiar docencia ${ }^{2}$.

Pero sí existe casi un $40 \%$ de personas graduadas de Arte Escénico que ejerce la docencia también; se puede decir que esta es una fuente laboral o una demanda y por ello, se plantea como una oferta. Siguiendo con los comentarios de las personas, estas mencionan que "hay docencia, todo mundo se inventa sus clases, pero una debe escribir proyectos para que le den trabajo"3.

¿Es posible decir que la docencia requiere también de la formulación de proyectos? o al menos y considerando que no existe en este país la formación formal en pedagogía teatral o la enseñanza del teatro, entonces $i$ se ha ido desarrollando en el camino la demanda?

Me di cuenta de la necesidad de investigar cómo articular una clase que tuviera una coherencia para niños de 7 a 10 años cuyos tiempos eran muy distintos a los de los adultos y que los ejercicios que yo había aprendido no estaban dirigidos a esa población. Entonces ahí empecé a investigar por mi propia cuenta todo lo que tenía que ver con dar clases, específicamente a niños ${ }^{4}$.

Las personas egresadas del Arte Escénico han desarrollado estrategias laborales también por la dificultad de desarrollarse en procesos

2 Palabras de Tatiana Chávez invitada a la mesa de diálogo, año 2017.

3 Palabras de Paola González, invitada de la mesa de diálogo y docente de la Escuela de Arte Escénico.

4 Ídem 
escénicos y artísticos; sin embargo, al empezar a desempeñarse en la docencia algunas personas optan por capacitarse en enseñanza.

Me interesaba la docencia por mi participación en el proyecto Teatro Aplicado y el Teatro de las Personas Oprimidas y cuando salí del bachillerato decidí entrar a la Escuela de Educación no Formal de la UCR, necesitaba un espacio que me pudiera dar herramientas que me podían hacer falta ${ }^{5}$.

Esa necesidad de herramientas o de buscar otros espacios indican el faltante dentro del plan de estudios de la escuela, pero también se asoma otro detalle: la participación en proyectos o programas de la EAE genera esas inquietudes. ¿Son los proyectos y programas de la EAE espacios que proyectan el posible perfil laboral de las personas egresadas de esta?

La oferta laboral de las personas egresadas en las Artes Escénicas tiene que ver, por ejemplo, con el sector cultura, Ministerio de Cultura y sus programas, municipalidades, centros culturales, entre otros, no relacionados con la interpretación o la actuación necesariamente, pero al entrar a participar en la oferta, la necesidad es el motor de la actualización profesional.

Esto también implica otras necesidades como la formulación de proyectos y por ello la actualización permanente es necesaria. "Me correspondió realizar el PAO (Plan Anual Operativo) en el Parque la Libertad, el plan de estudios del área de teatro para PAM (personas adultas mayor), pero descubrí la necesidad de aprender a redactar presupuestos, proyectos y vamos aprendiendo porque la vida se lo da"6.

Según Vargas (2012), "la falta de profesores de teatro en las aulas de primaria y secundaria en conjunto con la falta de formación en pedagogía teatral en las universidades son factores que al final del día pesan en la forma en que la población llega a apreciar al teatro" (p. 1), factores que no solamente influyen en la formación, especialización y perfiles laborales de la población egresada de la EAE, sino también en la generación de públicos de teatro.

5 Palabras de Paula Aguilar, participante de la mesa de diálogo y estudiante egresada de la Escuela de Arte Escénico.

6 Palabras de Adriana Zúñiga, participante de la mesa de diálogo y estudiante egresada de la Escuela de Arte Escénico 
Dentro del Ministerio de Educación Pública no existe el código de persona docente de teatro o arte escénico; por ello, tampoco es obligatorio especializarse en la enseñanza como sí lo es en las artes plásticas y la música, pero sí existen colegios y escuelas que ofrecen procesos teatrales. Un ejemplo claro es el Conservatorio Castella, el cual desde 1963 posibilita sesiones de teatro; otros tantos han iniciado ese camino e inclusive en la educación privada los clubes de teatro marcan una posibilidad de ingreso para la persona egresada de la EAE.

En la actualidad, existen cuatro espacios de formación de las artes escénicas: en el nivel de educación superior, técnica, educación básica y no formal en academias y centros artísticos/culturales, como la Escuela de Arte Escénico y la Escuela de Artes Dramáticas en el nivel superior; no muy lejana a las anteriores está el Taller Nacional de Teatro.

En el nivel superior también se encuentra lo no profesional, como lo son los grupos representativos de las cinco universidades estatales y los cursos de generales de la Universidad Nacional; en el nivel técnico y educación básica se ubican el ya mencionado Conservatorio Castella, Parque la Libertad, Colegio Técnico Artístico Profesor Felipe Pérez Pérez.

También, se encuentran academias como el Teatro Impromptu Giratablas, teatros en donde imparten talleres como el Moliere, Casa Nega, Casa 210, El Triciclo, Casa de la Ciudad, Centro Alajuelense de la Cultura, Teatro Sombrero Rojo, Talleres en TEC, Programa Teatro Aplicado, entre otros.

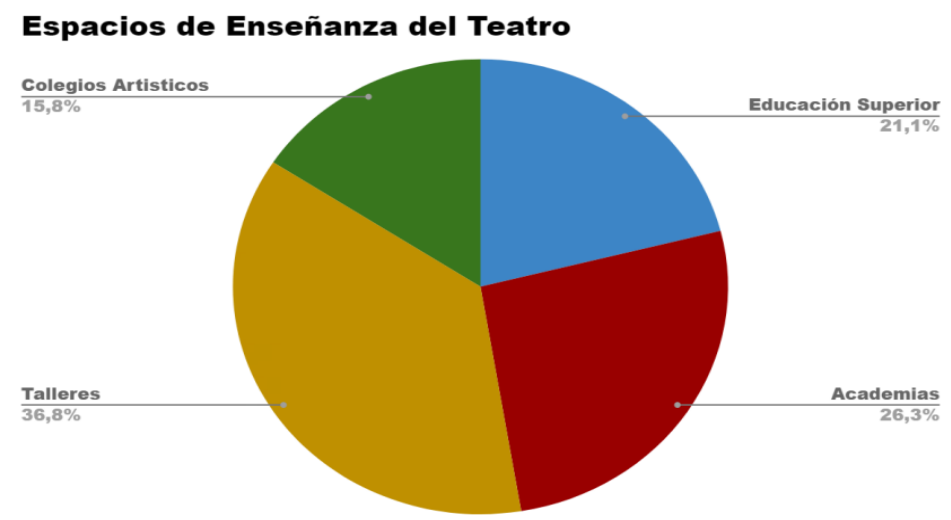

Figura 2. Espacios de enseñanza del teatro

Nota: elaboración propia. 
Son muchos los espacios en donde se está ejerciendo la enseñanza del teatro, "no estamos valorando cuál enseñanza del teatro, sino el cómo se está enseñando teatro" ". Estas palabras generaron un sentir en las personas participantes de la mesa, que además se han dedicado a la enseñanza de las Artes Escénicas. Sin embargo, no ha sido solamente en este espacio que se sigue reflexionando, sino que en otros se han iniciado procesos de reflexión-acción y de generación de propuestas y espacios como el proyecto Pre-Carrera de la Escuela de Arte Escénico.

Otros de los espacios actuales son: el Laboratorio de Pedagogía Teatral, dirigido por estudiantes egresadas de la Escuela de Artes Dramáticas de la Universidad de Costa Rica, que lleva actualmente el cuarto proceso; el curso de Pedagogía en la Escuela de Artes Dramáticas, las Primeras Jornadas de Reflexión sobre Pedagogía Teatral realizadas en el 2018 por la Escuela de Arte Escénico.

También existen espacios personales, en el marco de tesis de graduación y procesos investigativos académicos de la Escuela de Educación No formal de la Universidad de Costa Rica, por estudiantes egresadas tanto de la Escuela de Arte Escénico y la Escuela de Arte Dramáticas.

\section{¿Qué hace falta?}

Ferrater (1994, según se cita en Rivelis, 2013), recuerda que:

el silencio puede formar parte entonces del diálogo. Pero hay que distinguir entre el diálogo auténtico y el falso. El diálogo auténtico (implique o no comunicación por medio de palabras) es aquel en el cual se establece una relación viva entre personas como personas. El diálogo falso (calificado de "monólogo") es aquel en el cual los hombres creen que se comunican mutuamente cuando lo único que hacen en verdad es alejarse unos con otros [...]. (p. 34)

Las interrogantes siempre van a existir y también las respuestas; reconocer necesidades, urgencias y reflexiones es válido, pero el accionar o el cómo se hace lo es aún más. Lejos de dar una única lectura a la encuesta, abrimos la posibilidad de que toda persona que conoce la EAE y lea este escrito pueda también formular su opinión a la pregunta:

7 Palabras de Wendy Hall, coordinadora de la mesa de diálogo, egresada y docente de la Escuela de Arte Escénico. 
¿es la Escuela de Arte Escénico un espacio de educación pública superior que fomenta la formación de profesionales, capaces de incidir en la sociedad en ejes estratégicos como los son la investigación, la extensión y la producción nacional de teatro?

Podría ser que la persona que realiza esta lectura sea egresada de la EAE, funcionaria/docente actual, pedagoga o participante de un grupo de teatro independiente, que reconoce que en esta investigación hace falta escuchar más voces, realizar mayores profundizaciones del material, pero que también, si ha sentido a la EAE y como parte del gremio teatral, sabe del gremio teatral costarricense.

Hace falta hablar de temas que no se abordaron, como lo es el teatro independiente, otro de los porcentajes mayores; de las razones por las cuales las personas egresadas de la EAE son pocas y son escasas las matriculadas. Sin duda, como apunta Araque (2009): "Los que laboramos en el medio universitario y operamos por medio de modelos académicos, debemos contemplar las condiciones en las que llegan (y salen) los jóvenes a nuestras academias y sus posibilidades reales de formación" (p. 56).

La sistematización de este proceso de investigación quizá sea generadora de otros más que, ya sean para refutar, profundizar, detallar o bien aclarar, darán mayores insumos de lo que hasta ahora se conoce y reconoce del gremio teatral costarricense; no el que hace teatro, sino de aquel que ha atravesado al menos cuatro años en la EAE y ha reconocido en la escuela un espacio de formación.

\section{Referencias}

Araque, C. (2009). Razonamientos y desvaríos en torno a la pedagogía teatral. Revista Colombiana de las Artes Escénicas, 3, 53-69. Recuperado de http:/vip.ucaldas.edu.co/artescenicas/downloads/ artesescenicas3_7.pdf.pdf

Cerdas, D. (2014). Colección UNA extensión universitaria con arte. Escuela de Arte Escénico: Editorial EUNA.

Freire, P. y Faúndez, A. (2013). Por una pedagogía de la pregunta. Buenos Aires: Siglo Veintiuno Editores Argentina S. A.

Medina, J. y Cembranos, F. (2003). Grupos inteligentes: teoría y práctica del trabajo en equipo. Madrid: Popular España.

Rivelis, G. (2013). Es posible aprender a dialogar inteligentemente. Novedades Educativas, 272, 32-34. 
Universidad Nacional. (2019). Graduados por sede, facultad, escuela según año, sexo. Recuperado de https://www.registro.una. ac.cr/see/index.php/graduacion/110-cuadros-powerbi/graduacion/331-graduados-por-sede-facultad-escuela-segun-ano-sexo

Universidad Nacional. (2020). Matrícula de primer ingreso. Recuperado de https://www.registro.una.ac.cr/see/index.php/ matricula-de-primer-ingreso

Vargas, L. (2012). Teatro en Costa Rica: problemas de oferta y demanda. Recuperado de http://www.redcultura.com/php/Articulos1024.htm

Vásquez, C. (2009). Pedagogía teatral una propuesta teórico-metodológica crítica. Calle14, 3(3), 61-73. 